\title{
Predicting Required COVID-19 Vaccine Coverage and its Impact in Sierra Leone Using Mathematical models
}

\author{
Joseph A. L. Kamara
}

Fourah Bay College, University of Sierra Leone

Abdul A. Kamara ( zaak.hust167@gmail.com )

Fourah Bay College, University of Sierra Leone

\section{Sallieu K. Samura}

Fourah Bay College, University of Sierra Leone

\section{Research Article}

Keywords: COVID-19, Vaccine coverage, Mathematical models, Asymptomatic-symptomatic infection

Posted Date: July 2nd, 2021

DOI: https://doi.org/10.21203/rs.3.rs-665793/v2

License: (c) (1) This work is licensed under a Creative Commons Attribution 4.0 International License.

Read Full License

Version of Record: A version of this preprint was published at Archives of Clinical and Biomedical Research on January 1st, 2022. See the published version at https://doi.org/10.26502/acbr.50170228. 


\title{
Predicting Required COVID-19 Vaccine Coverage and its Impact in
} Sierra Leone Using Mathematical models

\author{
Joseph A.L. Kamara, Abdul A. Kamara, Sallieu K. Samura \\ Department of Mathematics and Statistic, Fourah Bay College, University of Sierra Leone, \\ Freetown, Sierra Leone
}

Corresponding author: Abdul A. Kamara; Email: zaak.hust167@gmail.com

\begin{abstract}
In this article, we predict the required vaccine coverage to eradicate the COVID-19 outbreak in Sierra Leone. We also, investigate the impact of facemask and vaccine coverage on the spread of the COVID-19 virus using a modified symptomatic-asymptomatic infection transmissions Susceptible-LatentInfectious-Asymptomatic-Recovered (SLIAR) model. We derived an explicit formula for the basic reproduction number and used it to understand the dynamics of the disease when it is greater than unity. We also used Maximum Likelihood modelling technique to estimate the basic reproduction number using incidence data. Numerically, we show that 58 per cent of the Sierra Leone national population required vaccination to eradicate the COVID-19 virus. Also, the SLIAR with vaccine model results reveal that the impact of using facemask is very challenging to understand and the vaccine coverage decrease the infected transmission rate but cannot completely eradicate the infection.
\end{abstract}

Keywords: COVID-19, Vaccine coverage, Mathematical models, Asymptomatic-symptomatic infection

\section{Introduction}

The 2019 coronavirus (COVID-19) outbreak has been global pandemic with over 140,000,000 cases and more than 3,000,000 deaths[1]. Because of its high rate of infection and mode of transmission many control strategies have been implemented like social distancing, use of face mask, handwashing, and vaccine. Facemask is used because the virus of the COVID-19 can transmit through sneezing and coughing[2, 3]. Regular handwashing because human frequently touches their face which is the fastest transition point of the virus[4]. Social distancing because the virus can spread between a distance less than $6.1 \mathrm{ft}[5]$. The fear imposed by the COVID-19 is that people without symptom whether they can show symptoms or not, as 
long as they have exposed to the virus they can cause transmission[6-9]. That is, there is an asymptomatic form of transmission.

Although the recovered rate of the COVID-19 is encouraging, the deaths rate in most developed nations is alarming and because of that, the use of the vaccine has been the focus by many developed nations to eradicate the COVID-19 virus. Many vaccines have been used which developing countries like Sierra Leone is also a beneficiary. In February 2021, the People Republic of China donated 200,000 doses of vaccine [10] and another 96,000 doses of the AstraZeneca-Oxford COVID-19 vaccine shipped via the COVAX facility was donated on March 8, 2021, [11] help to fight the COVID-19 virus in Sierra Leone. The targeted populace are frontline health workers (18 years and above), vulnerable including aged people, 30 years and above healthy individuals and people with underlying health conditions[11, 12].

However, whilst many countries are preventing the third wave of the COVID-19, there is concern among public health officials that Sierra Leone is on the verge of a third wave of COVID-19 infections [12]. New cases are surging and have hit highs record since June 1, 2021 [12]. Like many African countries, citizens in Sierra Leone have been reluctant to take the COVID-19 vaccine. As of June 16, 2021, estimated vaccine total doses given is 91,789 and people fully vaccinated is 13,831 which is about 0.2 per cent of Sierra Leone populations [13]. Among the recent restriction by the Sierra Leone government as of June 17, 2021 are; age for vaccination has been revised further to thirty (30) years and educational institutions shall comply with mandatory face mask, hand-washing and social distance protocols [12]. As a researcher, our main concern is the fraction of the population that must be successfully vaccinated to eradicate the infectious agent so that the government policy which states that government ministries, departments and agencies will only be accessed by employees and members of the public with proof of at least one dose of COVID-19 vaccination[12] can be revisited.

A mathematical models has been an effective tool to understand disease dynamics[14]. The state compartments in the system are demonstrated using differential equations from which an expression of the basic reproduction number can be derived[14]. The basic reproduction number $\left(R_{0}\right)$ is a value that determines on average how many individuals an infected person infect during the infectious period. If $R_{0}>$ 1 , it believes that the disease will persist and becomes endemic and if $R_{0}<1$, the disease will die out and the system becomes disease-free[15]. After the $R_{0}$ has been determined, the basic reproductive number under vaccination which is the number of secondary cases caused by one primary case introduced into a population in which a proportion have been vaccinated [16] can also be determined. Also, the critical vaccination proportion that will achieve the eradication of the virus, for which the basic reproductive number under vaccination is equal to unity [16] can be determined. 
In this article, we modified a symptomatic-asymptomatic infection transmission SusceptibleLatent-Infectious-Asymptomatic-Recovered (SLIAR) model for Influenza outbreak with a vaccine (V), used of facemask and handwashing. The symptomatic-asymptomatic infection transmission SLIAR model is common in the literature of SARS-CoV-1 $[17,18]$ due to its mode of transmission as it is done in COVID19. That is why scientist referred to the COVID-19 as SAR-CoV-2[19, 20] as they get to know more about the COVID-19 virus. We aim to use the mathematical SLIARV model to inspect the impact of facemask, and vaccine coverages using epidemic parameters in Sierra Leone. We also, aim to use Maximum Likelihood modelling [21] to estimate the $R_{0}$ from Sierra Leone daily reported incidence data and use it to estimate the proportion of people to vaccinate in other to eradicate the SARS-CoV-2 in Sierra Leone. By considering the vaccine coverage period in Sierra Leone, the estimated $R_{0}$ is 2.4 showing that 58 per cent of the national population required to vaccinate to eradicate the COVID-19 virus. Also, our mathematical model results shows that the impact of using facemask is very challenging to understand and the vaccine coverage decrease the infected transmission rate but cannot completely eradicate the infection.

\section{The model}

In this article, we consider a varying population SLIARV model since the SARS-CoV-2 has been going on for more than a year and there is no restriction of movement in and out of Sierra Leone during the time of writing. The model is demonstrated using the differential equation given as

$$
\begin{aligned}
& \frac{d S}{d t}=\Lambda N-\frac{\beta S}{N}(I+\varepsilon A)\left(1-\lambda_{1}\right)\left(1-\lambda_{2}\right)-(\mu+p) S, \\
& \frac{d L}{d t}=\frac{\beta S}{N}(I+\varepsilon A)\left(1-\lambda_{1}\right)\left(1-\lambda_{2}\right)-(\mu+\kappa) L, \\
& \frac{d I}{d t}=\eta \kappa L-\left(\mu+d+r_{2}\right) I \\
& \frac{d A}{d t}=\kappa(1-\eta) L-\left(\mu+r_{1}\right) A \\
& \frac{d R}{d t}=r_{1} A+r_{2} I+r_{3} V-\mu R \\
& \frac{d V}{d t}=p S-\left(\mu+r_{3}\right) V
\end{aligned}
$$

Where $S=S(t)$ is the susceptible, $L=L(t)$ is the latent, $I=S(t)$ is the symptomatically infection, $A=$ $A(t)$ is the asymptomatically infection, $R=R(t)$ recovered and $V=V(t)$ vaccination populations at time $t$. The total population is $N=S+L+I+A+R+V, \Lambda$ is the rate of birth/migration into the $\mathrm{S}$ population, 
$\beta$ is the symptomatic infective transmission coefficient and the infectiveness due to an asymptomatic person is reduced by the proportion $\varepsilon$. The rate of facemask and handwashing are denoted as $\lambda_{1}$ and $\lambda_{2}$ respectively, whereas $\mu$ and $p$ are the natural death rate and proportion of vaccinated individual respectively. The incubation period rate is measure by $\kappa$ and the probability of being symptomatic infective is measure by $\eta$. Death related to the disease is measure by the rate $d$, the recovered rate from populations of $A, I$ and $V$ are respectively denoted as $r_{1}, r_{2}$, and $r_{3}$. For simplicity equations (1) to (6) is converted to proportional form given as

$$
\begin{aligned}
& \frac{d s}{d t}=\Lambda-\beta s(i+\varepsilon A)\left(1-\lambda_{1}\right)\left(1-\lambda_{2}\right)-(\mu+p) s, \\
& \frac{d l}{d t}=\beta s(i+\varepsilon a)\left(1-\lambda_{1}\right)\left(1-\lambda_{2}\right)-(\mu+\kappa) l, \\
& \frac{d i}{d t}=\eta \kappa l-\left(\mu+d+r_{2}\right) i, \\
& \frac{d a}{d t}=\kappa(1-\eta) l-\left(\mu+r_{1}\right) a, \\
& \frac{d v}{d t}=p s-\left(\mu+r_{3}\right) v,
\end{aligned}
$$

Where $s=S / N, l=L / N, i=I / N, a=A / N, v=V / N$, and equation (6) is excluded for further analysis since it does not influence the other equations. It easy to see that if $\lambda_{1}=\lambda_{2}=p=0$, equations (7) to (11) will be in the same form as the influenza model in [18]. However, it is not difficult to see that the feasible region of the model (2)

$$
\Gamma=\left\{(s, l, i, a, v) \in R_{+}^{5}: s+l+i+a+v \leq \frac{\Lambda}{\mu+p}\right\}
$$

is positive invariant and attracting sets that attracts all solutions of model (2) with nonnegative initial condition. Also, it is not difficult to see that model (2) has disease-free equilibrium (DFE), $E_{0}=$ $\left(s^{0}=\Lambda /(\mu+p), 0,0,0,0\right)$ and using the next-generation matrix method [15]A we get

$$
R_{0}=\frac{\eta \Lambda \kappa \beta\left(1-\lambda_{1}\right)\left(1-\lambda_{2}\right)}{(\mu+p)(\mu+\kappa)\left(\mu+d+r_{2}\right)}+\frac{\Lambda \kappa \beta \varepsilon(1-\eta)\left(1-\lambda_{1}\right)\left(1-\lambda_{2}\right)}{(\mu+\kappa)(\mu+p)\left(\mu+r_{1}\right)}
$$

It is well known that the disease will die out whenever $R_{0}<1$ or continue to persist in the population whenever $R_{0}>1$. For a perfect vaccine that confers life-long protection, the basic reproductive number 
under vaccination $\left(R_{0 p}\right)$ can be determined as $(1-p) R_{0}$, and the vaccination proportion that will achieve eradication $\left(P_{c}\right)$ is determine by $\left(1-1 / R_{0}\right)[16]$.

\section{Numerical simulations}

In this section, we use R-software to run simulations for our SLIAR-V model and the values of the parameters are taken from epidemiological data for Sierra Leone. In Sierra Leone as of June 20, 2021, the average number of handwashing facilities is 19.275[22] and 1.4 per 10000 healthcare workers as of 2016 [23]. Because healthcare workers frequently wash their hands, we estimated $\lambda_{2}$ as the ratio of average handwashing facilities and average healthcare workers. Sierra Leone has administered at least 91,789 doses of COVID vaccine as of June 16,2021 , which is $0.6 \%$ of the country's population if assuming every person needs two doses to be fully vaccinated [13]. Therefore, our assumed initial estimate of $p=0.006$ and also, since there is no report of partial vaccination, we assumed all those who are vaccinated move to the recovery state with permanent immunity. The lifespan of Sierra Leone as of 2019 is 54.7 years[24], hence $\mu=$ $1 /(54.7 \times 365)$ days. There are 82 deaths out of 4816 infected cases as of June 20, 2021[25], therefore we estimate deaths related to the disease as $d=82 / 4816$. The incubation period is 14 days and the recovery period from visibly infectious has an average of 5.8 days [26]. We also assume the rate of moving from an asymptomatic state to recovery rate to be 14 days since it is the same observed period use for quarantine individual. Table 1 gives the value of the parameter used in the numerical simulation,

Table 1. The Sierra Leone demography and epidemiological parameter values

\begin{tabular}{|c|l|l|l|}
\hline Parameters & Description & Values & Source \\
\hline$\lambda_{1}$ & Facemask & 0.001 & Assumed \\
\hline$\lambda_{2}$ & Handwashing & 0.001 & {$[22]$} \\
\hline$p$ & Vaccinated proportion & 0.006 & {$[13]$} \\
\hline$\varepsilon$ & Latency transmission efficacy & 0.5 & {$[14]$} \\
\hline$\mu$ & Natural death rate & 0.0005 & {$[24]$} \\
\hline$d$ & Death related to disease & 0.02 & Calculated \\
\hline$\kappa$ & Incubation period & 0.07 & {$[26]$} \\
\hline$r_{1}$ & Asymptomatic recovery & 0.07 & Assumed \\
\hline$r_{2}$ & Visibly infectious recovery & 0.712 & Assumed \\
\hline$r_{3}$ & Vaccinated recovery & 0.006 & Assumed \\
\hline$\Lambda$ & Birth rate & 0.0042 & {$[27]$} \\
\hline$\eta$ & Symptomatic infective rate & 0.05 & Assumed \\
\hline$\beta$ & Transmission rate & 0.533 & {$[14]$} \\
\hline
\end{tabular}

Using the parameter values from Table 1 , we get $R_{0}=2.4$, which is the $R_{0}$ values for Sierra Leone, when simulating the incidence data from January 1, 2021, to June 20, 2021. Using an Maximum Likelihood modelling as described in [21] our estimated $R_{0}=2.4$ with confidence interval $(2.248304,2.670513)$. The 
estimate of the basic reproductive number under vaccination is then $(1-p) R_{0}=(1-0.006) .(2.4)=$ 2.4. However, the critical vaccination proportion $\left(P_{c}\right)$ that will achieve eradication of the virus, for which the basic reproductive number under vaccination is equal to unity is determine as $1-1 / R_{0}=1-0.42=$ 0.58. That is, based on the current transmission rate, if approximately $58 \%$ of the Sierra Leone population is vaccinated, eliminating the COVID-19 will not be a challenge.

Furthermore, we now focus our analysis on the impact of the control measured $\left(p, \lambda_{1}, \lambda_{2}\right)$ considered in the SLIAR vaccine model. Firstly, we simulate the SLIAR-V model without any adjustment of the values of the parameters in Table 1 to observe the behaviour of the individual population trajectory, especially the visibly infectious compartment. It is observed in Figure.1A that the symptomatic infected trajectory increases with increasing time despite the use of facemask, handwashing and vaccine parameters. Also, it observes that when $\boldsymbol{I}$ and $\boldsymbol{A}$ trajectories increase the susceptible decreases, indicating their negative effect to the susceptible populations if they are not controlled.
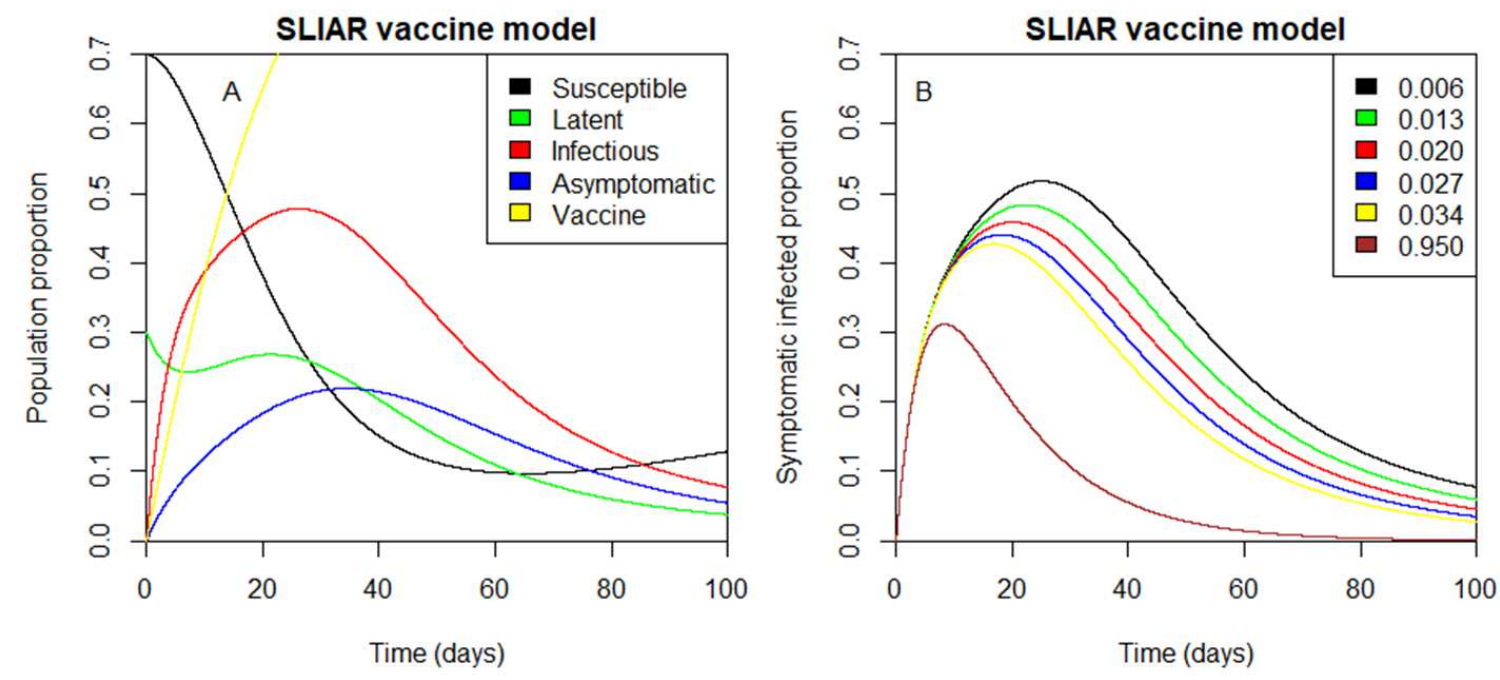

Figure 1 Numerical simulations for the COVID-19 SLIAR vaccine model behaviour when the model is unchanged (A) and when the vaccination increases (B). Vaccination refers to the percentage of susceptible individual vaccinated.

Figure $1 \mathrm{~B}$ show the impact of increasing the vaccination rate constant by $0.7 \%$. It is well known that when vaccine coverage is large and immunity is lifelong, the prevalence of infection decreases. This is observed in Figure 1B as the vaccination rate increases. The trajectories peaks of the symptomatic infected proportion decrease but it does not reach zero even at 95\% coverage. Moreover, even though the vaccine coverage increases equally with facemask and handwashing coverages (Figure 2A), the symptomatic infected proportion trajectories peaks decrease but do not reaches zero. This shows that large vaccine 
coverage reduces the infection spread, but does not lead to complete eradication of the disease as demonstrated in [28]. Thus, the strategy of controlling COVID-19 by vaccination needs to be improved in terms of vaccinating the younger ages of an individual to tackle the infection.
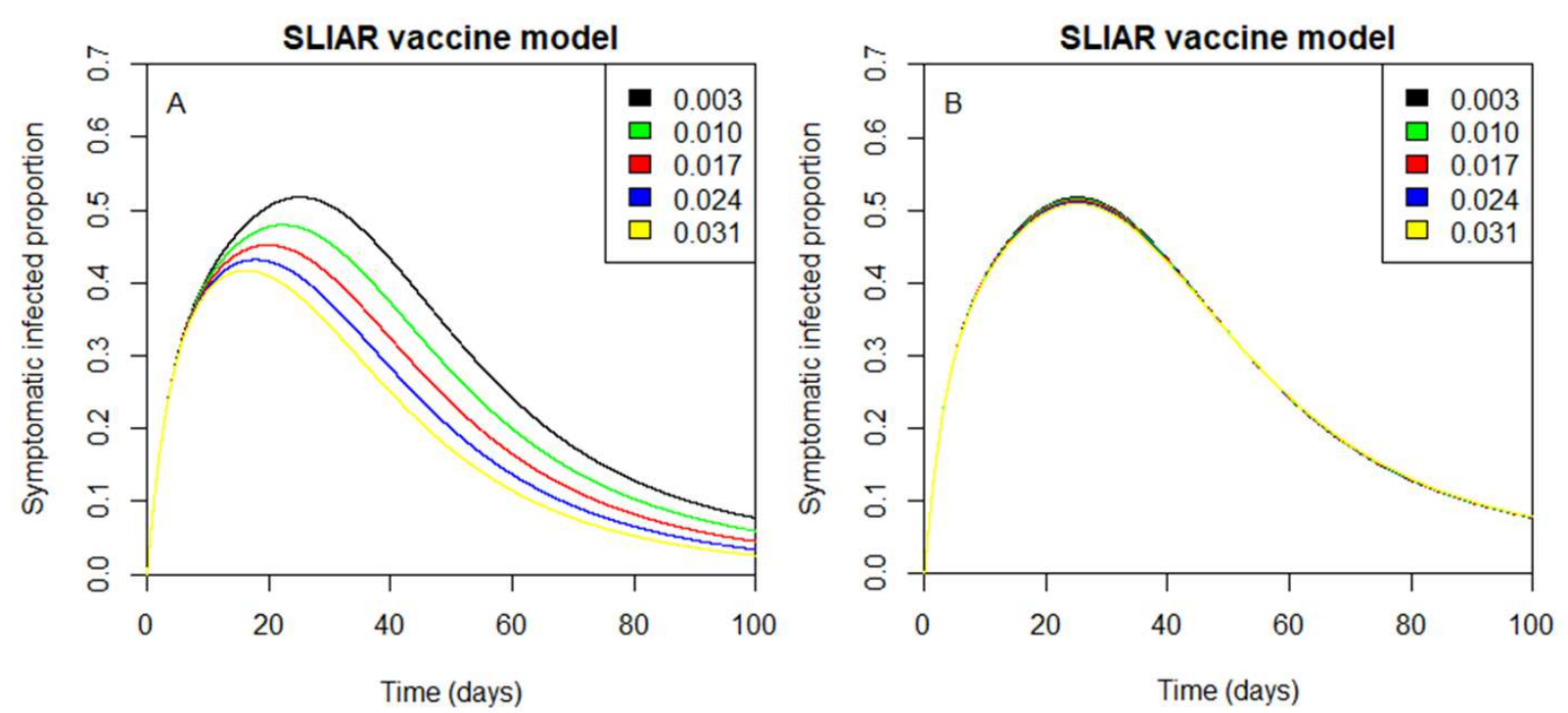

Figure 2 Numerical simulation for the SLIAR vaccine model when the vaccinating, facemask and handwashing (A) increase equally by $0.7 \%$, and (B) is when all parameters are remaining unchanged as given in Table 1 and increase the magnitude of the facemask coverage by $0.7 \%$.

In Figure 2B, it is observed that despite the $0.7 \%$ increment of the facemask coverage the same trajectories are produced and it is difficult to give an insightful characterization of the epidemic curve. This shows that there is a problem of identifying the importance of using facemask with the symptomaticasymptomatic infections SLIAR vaccine model.

\section{Discussion}

This paper aims to extend a basic symptomatic-asymptomatic infection SLIAR model without control of Influenzas propagation $[17,18]$ to model the used of facemask, handwashing and vaccination as a control strategy for COVID-19 pandemic. We investigate the effects of the facemask and vaccination rate on the spread of the coronavirus infection in Sierra Leone. We have derived an explicit formula for the basic reproduction number $\left(R_{0}\right)$, which has been the key parameter in our model. It is well known that when $R_{0}>1$, there exists a unique endemic equilibrium, which indicate that the disease is a treat in the population. Numerical simulations are conducted to illustrate that the infection maintain in the population with time when $R_{0}$ is great than unity (see Figure $1 \mathrm{~A}$ ). 
In terms of calculating $R_{0}$, we show that the $R_{0}$ estimated from the SLIAR vaccine model is equivalent to the current estimated $R_{0}$ for Sierra Leone COVID-19 outbreak using Maximum Likelihood modelling[21]. It shows that from January 1,2021 , to June 20, 2021, the estimated $R_{0}$ is 2.4 , indicating that an infectious person can infect an average of two persons in his/her infectious period. Approximately, our estimated $R_{0}$ is in agreement with reproduction rate of Our World in Data[22]. Because the transmission rate is alarming in Sierra Leone, we estimated the critical vaccination proportion of people to achieve the eradication of the virus as $58 \%$, which is far above the vaccine program focus targeted population in Sierra Leone. According to the World Population Prospects 2019, Volume II [24], there were 39\% of the country population between the age groups 25 years to 65 years and above. Therefore, targeting 18 years and above health professionals which majority are above aged 25 years, and the aged 30 years, for the vaccine coverage in Sierra Leone will impose a serious challenge to eradicate the COVID-19 disease outbreak. Hence, the policy of vaccinating just portion of the susceptible population needs to be revisited by including young ages of an individual to tackle the COVID-19 in Sierra Leone. The predicting vaccine coverage in this article can only be reach if the targeted population can be reduce to at least age 15 years using the World Population Prospects 2019, Volume II data[24]

Using the SLIAR vaccine model the vaccination coverage only involves those in the susceptible population. In terms of controlling the spread of COVID-19 by facemask, we show that the importance of using facemask cannot be identified, as the same trajectory produce each time the coverage proportion increases. By controlling the spread of the COVID-19 virus by vaccination, we also show that even though vaccination reduces the infection spread, it does not completely eradicate the COVID-19 in the community when the coverage is high which is in agreement with [28].

\section{Declarations}

Availability of data materials. Authors can confirm that all relevant data sources are included in the article.

Competing interests. The authors declare that they have no competing interests,

Funding: Not applicable.

\section{References}

1. JHU, J.H.U.: Coronavirus COVID-19 Global Cases by the Center for Systems Science and Engineering (CSSE)

2. CDC, C. for D.C. and P.: How COVID-19 Spreads, https://www.cdc.gov/coronavirus/2019ncov/prevent-getting-sick/how-covid-spreads.html 
3. WHO, W.H.O.: How COVID-19 Spreads, https://www.who.int/news-room/q-a-detail/coronavirus\%0Adisease-covid-19-how-is-it-transmitted

4. CDC, C. for D.C. and P.: Transmission of Coronavirus Disease 2019 (COVID-19). CDC Bull. (2020)

5. WHO, W.H.O.: Coronavirus disease (COVID-19): How is it transmitted?, https://www.who.int/news-room/q-a-detail/q-a-how-is-covid-19-transmitted, (2020)

6. Anastassopoulou, C., Russo, L., Tsakris, A., Siettos, C.: Data-based analysis, modelling and forecasting of the COVID-19 outbreak. PLoS One. 3, 1-21 (2020).

https://doi.org/10.1371/journal.pone.0230405

7. Furukawa, N.W., Furukawa, N.W., Brooks, J.T., Sobel, J.: Evidence Supporting Transmission of Severe Acute Respiratory Syndrome Coronavirus 2 while Presymptomatic or Asymptomatic. Emerg. Infect. Dis. 26, (2020). https://doi.org/10.3201/eid2607.201595

8. Li, C., Ji, F., Wang, L., Wang, L., Hao, J., Dai, M., Liu, Y., Pan, X., Fu, J., Li, L., Yang, G., Yang, J., Yan, X., Gu, B.: Asymptomatic and Human-to-Human Transmission of SARS-CoV-2 in a 2Family Cluster, Xuzhou, China. Emerg. Infect. Dis. 26, (2020).

https://doi.org/10.3201/eid2607.200718

9. $\quad$ Kimball, A., Hatfield, K.M., Arons, M., James, A., Taylor, J., Spicer, K., Bardossy,et al.: Asymptomatic and Presymptomatic SARS-CoV-2 Infections in Residents of a Long-Term Care Skilled Nursing Facility — King County, Washington, March 2020. MMWR. Morb. Mortal. Wkly. Rep. (2020). https://doi.org/10.15585/mmwr.mm6913e1

10. Xinhua: China donates 200,000 doses of COVID-19 vaccine to Sierra Leone, http://www.xinhuanet.com/english/2021-02/26/c_139767646.htm, (2021)

11. WHO, W.H.O.: COVID-19 vaccines shipped by COVAX arrive in Sierra Leone, https://www.afro.who.int/news/covid-19-vaccines-shipped-covax-arrive-sierra-leone, (2021)

12. Mic.gov.sl: $(20+)$ The Ministry of Information and Communication _ Facebook, https://www.facebook.com/mic.gov.sl/, (2021)

13. Reuters: Sierra Leone: the latest coronavirus counts, charts and maps, https://graphics.reuters.com/world-coronavirus-tracker-and-maps/countries-and-territories/sierraleone/, (2021) 
14. Kamara, A.A., Mouanguissa, L.N., Barasa, G.O.: Mathematical modelling of the COVID-19 pandemic with demographic effects. J. Egypt. Math. Soc. 29, (2021).

https://doi.org/10.1186/s42787-021-00118-7

15. Van Den Driessche, P., Watmough, J.: Reproduction numbers and sub-threshold endemic equilibria for compartmental models of disease transmission, (2002)

16. Nikbakht, R., Baneshi, M.R., Bahrampour, A.: Estimation of the basic reproduction number and vaccination coverage of influenza in the United States (2017-18). J. Res. Health Sci. 18, 1-6 (2018). https://doi.org/10.34172/jrhs184239

17. Arino, J., Brauer, F., Van Den Driessche, P., Watmough, J., Wu, J.: Simple models for containment of a pandemic. J. R. Soc. Interface. 3, 453-457 (2006).

https://doi.org/10.1098/rsif.2006.0112

18. Lamichhane, S., Chen, Y.: Global asymptotic stability of a compartmental model for a pandemic. J. Egypt. Math. Soc. 23, 251-255 (2015). https://doi.org/10.1016/j.joems.2014.04.001

19. Du, Z., Xu, X., Wu, Y., Wang, L., Cowling, B.J., Meyers, L.A.: Serial Interval of COVID-19 among Publicly Reported Confirmed Cases. Emerg. Infect. Dis. 26, (2020).

https://doi.org/10.3201/eid2606.200357

20. Health, W.H.O., Programme, E., Panel, E.A., Preparedness, I.P.C., Guidance, I.P.C., Group, D., Gdg, I.P.C., Preparedness, S., Plan, R.: Transmission of SARS-CoV-2 : implications for infection prevention precautions, https://www.who.int/news-room/\%0Acommentaries/detail/transmissionof-sars-cov-2-implications-for-infection-prevention-\%0Aprecautions, (2020)

21. Obadia, T., Haneef, R., Boëlle, P.Y.: The R0 package: A toolbox to estimate reproduction numbers for epidemic outbreaks. BMC Med. Inform. Decis. Mak. 12, (2012). https://doi.org/10.1186/1472-6947-12-147

22. Owid: covid-19-data_public_data at master · owid_covid-19-data $\cdot$ GitHub, https://github.com/owid/covid-19-data/tree/master/public/data, (2021)

23. Robinson, C.: Primary health care and family medicine in Sierra Leone. African J. Prim. Heal. Care Fam. Med. 11, 1-3 (2019). https://doi.org/10.4102/phcfm.v11i1.2051

24. UNDESAPD, U.N.D. of E. and S.A.P.D.: World Population Prospects 2019: Demographic Profiles. (2019) 
25. Worldometer: Sierra Leone COVID_4,042 Cases and 79 Deaths, (2021), https://www.worldometers.info/coronavirus/

26. Li, Q., Guan, X., Wu, P., Wang, X., Zhou, L., Tong, Y., Ren, R., Leung, K.S.M.,et al.: Early Transmission Dynamics in Wuhan, China, of Novel Coronavirus-Infected Pneumonia. N. Engl. J. Med. (2020). https://doi.org/10.1056/NEJMoa2001316

27. SSL, S.S.L.: Sierra Leone Demographic and Health Survey 2019 key indicators. (2019)

28. Nkamba, L.N., Manga, T.T., Agouanet, F., Manyombe, M.L.M.: Mathematical model to assess vaccination and effective contact rate impact in the spread of tuberculosis. J. Biol. Dyn. 13, 26-42 (2019). https://doi.org/10.1080/17513758.2018.1563218 